\title{
Truncated Conjugate Gradient Method for History Matching in Reservoir Simulation
}

\author{
Flávio Dickstein \\ Instituto de Matemática, UFRJ \\ Paulo Goldfeld \\ Instituto de Matemática, UFRJ \\ Gustavo Pfeiffer \\ Institute of Industrial Science, the University of Tokyo \\ Renan Pinto \\ LabMAPetro, UFRJ
}

\begin{abstract}
History Matching (HM) is an important problem in Oil Reservoir Simulation. We present here the Truncated Conjugate Gradient (TCG) Method to solve this problem. We compare TCG with two other well known schemes, TSVD and L-BFGS, in a numerical experiment using the benchmark problem PUNQ-S3. Our results indicate that TCG is a valuable tool for HM.

key-words Oil Reservoir Simulation, History Matching, Truncated Conjugate Gradient.
\end{abstract}

\section{Introduction}

Simulations of multiphase flows in porous media are the "nuts and bolts" of petroleumreservoir engineers. A reservoir simulator is the piece of software that computes, for instance, the evolution of oil and gas production rates or bottom-hole pressure at each well, by means of numerically solving the appropriate partial differential equations. See [9].

A reservoir simulator takes as input a model of the reservoir: a description of its geometry, of the properties of the rock and of the fluids, etc. Such data are not readily available for natural reservoirs (often inconveniently buried under miles of water, rock and salt), but rather obtained indirectly, from seismic data or geological knowledge about the formation. Using this a priori information, an exploitation strategy for the reservoir is conceived and implemented. After some years, the history of the field (past production data at wells) is often quite distant from what had been predicted by simulations based on the prior information.

History Matching (HM) is a data-assimilation technique, in which the history is used in order to improve the quality of the reservoir characterization. The basic idea is to look for reservoir parameters (such as permeability or porosity) for which simulation better matches the historical data actually recorded.

Various schemes have been designed to solve the HM problem, such as L-BFGS $[3,13]$ and TSVD $[1,10,12]$. Most recently, we have proposed the Truncated Conjugate Gradient 
(TCG) algorithm for HM [2]. As we describe in this work, TCG is based on the GaussNewton method. At each step, the optimization problem is linearized around the current approximation and the corresponding linear system is solved using the Conjugate Gradient method. As is usually the case with ill-posed problems, some regularization must be introduced. Here, this is done through a truncation procedure. We compare the behavior of TCG, TSVD and L-BFGS in some numerical experiments. In our results, TCG clearly outperforms TSVD. TCG displays a higher convergence rate than L-BFGS, although the latter runs faster.

The rest of this paper is organized as follows. In Section 2 we present a Bayesian formulation for the HM problem. In Section 3 the TCG algorithm is discussed. We recall the formulations of L-BFGS and TSVD in Section 4. Section 5 portrays a comparative study of the behavior of TCG, L-BFGS, TSVD in the PUNQ-S3 reservoir model [6]. This is a well known benchmark problem in HM.

\section{Mathematical Model}

Following [8], we assume that the a priori model is a Gaussian random variable whose probability distribution function (pdf) is given by

$$
f_{\text {prior }}(m) \propto \exp \left(-\frac{1}{2}\left\|m-m_{\text {prior }}\right\|_{C_{M}^{-1}}^{2}\right) .
$$

Here, $m_{\text {prior }}$ is the a priori mean, $C_{M}$ is the a priori covariance matrix and, for a symmetric positive-definite matrix $A,\|\cdot\|_{A}$ stands for the norm $\|m\|_{A}=\langle A m, m\rangle^{1 / 2}$.

Usually, the a priori information is rather poor. Therefore, the true reservoir production is often far from the prediction. In History Matching, one uses this new information, the actual history of the reservoir production (oil, gas and water productions, bottom-hole pressures at the wells, etc.) to better characterize the reservoir.

We consider that the vector $\epsilon$ of measurement errors of the history data $d_{o b s}$ is a normal random variable with covariance $C_{D}$. Further assuming that $\epsilon$ is independent of the a priori model, the a posteriori distribution (conditioned to the history data) satisfies

$$
f_{m \mid d_{o b s}}(m) \propto \exp \left(-\frac{1}{2}\left(\left\|m-m_{\text {prior }}\right\|_{C_{M}^{-1}}^{2}+\| g(m)-d_{o b s}\right) \|_{C_{D}^{-1}}^{2}\right) .
$$

Here $g(m)$ is the vector of observations associated with model $m$ (i.e., g represents the reservoir simulator) and $d_{o b s}$ is the vector of the actual observations, see [8]. One would like to generate a set of reservoir models sampling $f_{m \mid d_{o b s}}$. This is, in general, too costly. A less expensive procedure is to obtain the Maximum a Posteriori (MAP) solution

$$
m_{\text {map }}=m_{\text {map }}\left(m_{\text {prior }}, d_{\text {obs }}\right)=\arg \min _{m} J(m)
$$

where

$$
J(m)=\left\|m-m_{\text {prior }}\right\|_{C_{M}^{-1}}^{2}+\left\|g(m)-d_{o b s}\right\|_{C_{D}^{-1}}^{2} .
$$


A set of solutions to the problem can be obtained from the Randomized Maximum Likelihood (RML) strategy $[4,8]$. RML amounts to generating a set of $n$ vectors of observations $\left\{d_{o b s}^{(i)}\right\}_{i=1, \ldots, n}$ and of models $\left\{m_{\text {prior }}^{(i)}\right\}_{i=1, \ldots, n}$ according to the respective probability distributions and to obtain MAP solutions $m_{\text {map }}^{(i)}=m_{\text {map }}\left(m_{\text {prior }}^{(i)}, d_{\text {obs }}^{(i)}\right)$ of (1). In this way, the use of RML reinforces the need of solving the optimization problem (1) efficiently.

As is usual with inverse problems, HM is ill-conditioned. A regularizing procedure is to consider the Cholesky decomposition $C_{M}=L L^{T}$ and the change of variables

$$
\theta(m)=L^{-1}\left(m-m_{\text {prior }}\right), \quad m(\theta)=m_{\text {prior }}+L \theta,
$$

so that $\left\|m-m_{\text {prior }}\right\|_{C_{M}^{-1}}=\|\theta\|$. The MAP problem in $\theta$ is to obtain $\theta_{\text {map }}$ such that

$$
\theta_{\text {map }}=\underset{\theta}{\arg \min } J^{\theta}(\theta)
$$

where $J^{\theta}(\theta(m))=J(m)$, i.e., $J^{\theta}(\theta)=\|\theta\|^{2}+\left\|g(m(\theta))-d_{o b s}\right\|_{C_{D}^{-1}}^{2}$.

\section{TCG}

We now describe briefly the Truncated Conjugate Gradient (TCG) scheme to solve (4). More details can be found in [2]. We use Gauss-Newton, so that at each step $g(m(\theta))$ is replaced by its linearization around the current estimate $\theta_{k}$, i.e., $g(m(\theta)) \approx g_{k}+G_{k} L \Delta \theta$, where $g_{k}=g\left(m_{k}\right), m_{k}=m\left(\theta_{k}\right), \Delta \theta=\theta-\theta_{k}$ and $G_{k}=(d / d m) g\left(m_{k}\right)$, the derivative of the observations with respect to the model parameters, is the so-called sensitivity matrix. We thus obtain the linear least squares problem

$$
\min _{\Delta \theta} J_{k}^{\theta}(\Delta \theta)
$$

where $J_{k}^{\theta}(\Delta \theta)=\left\|\theta_{k}+\Delta \theta\right\|^{2}+\left\|G_{k} L \Delta \theta-r_{k}\right\|_{C_{D}^{-1}}^{2}$, with $r_{k}=d_{o b s}-g_{k}$. Then (5) is equivalent to solving

$$
\left(I+L^{T} G_{K}^{T} C_{D}^{-1} G_{k} L\right) \Delta \theta_{k}=L^{T} G_{k}^{T} C_{D}^{-1} r_{k}-\theta_{k} .
$$

Notice that $L^{T} G_{k}^{T} C_{D}^{-1} r_{k}-\theta_{k}=-\nabla J_{k}^{\theta} / 2$ and $I+L^{T} G_{K}^{T} C_{D}^{-1} G_{k} L=H_{k} / 2$, where $H_{k}$ is the Hessian of $J_{k}^{\theta}$. We apply the standard conjugate gradient (CG) algorithm (see, e.g., [5]) to solve (6). However, similarly to what happens with the TSVD algorithm, one has to appropriately truncate the CG iteration. Finally, the solution obtained using TCG provides a direction in which a line search is performed. It is possible to implement TCG without having to compute the Cholesky decomposition of $C_{M}$. This is relevant for large-scale problems, since the factorization cost scales with the cube of the grid size.

\section{$4 \quad$ TVSD and L-BFGS}

We briefly describe TSVD and L-BFGS, which have been previously employed in HM, see $[1,3,10,12,13]$. 
Analogously to TCG, TSVD is also based on Gauss-Newton. As with TCG, at step $k$ one has to solve (6). The solution can be obtained as a linear combination of the right singular values of $C_{D}^{-\frac{1}{2}} G_{k} L$. In TSVD, the summation in this linear combination is truncated in an appropriate way, see $[1,10,12]$ for further details.

The BFGS iterative method to solve the general problem of minimizing a function $f(x)$ was proposed simultaneously in 1970 by Broyden, Fletcher, Goldfarb and Shanno. The central idea is to construct at step $k$ a search direction $\Delta x_{k}$ using a rank-two update of the approximation $H_{k}$ of $f^{\prime \prime}\left(x_{k}\right)$. The limited-memory L-BFGS(n) scheme uses only the information of the $n$ most recent steps to build $H_{k}$. For more details, see $[4,7]$.

\section{$5 \quad$ Numerical Experiments}

We present numerical experiments comparing the performance of TCG, TSVD and L-BFGS for the HM problem. We observe that at each iteration TCG and TSVD approximately solve (6). This resolution involves the application of both the sensitive matrix $G_{k}$ and of its adjoint $G_{k}^{T}$ to vectors. In L-BFGS, the approximation of the Hessian is based solely on gradient values at previous iterates and therefore only applications of $G_{k}^{T}$ are required. In our experiments, we make use of SIMPAR, a basic fully implicit 3D black-oil simulator developed by Petrobras and featuring tools for the computation of derivatives and adjoints.

We considered the benchmark PUNQ-S3 [6] for our tests. It is a model with a grid size of $19 \times 28 \times 5$ and 1,761 active blocks. Figure 1 depicts a colormap of the depth of the reservoir top. The initial oil-water contact (blue contour) and gas-oil contact (red contour), a sealing fault (black line) and six production wells (black dots) are also indicated. There is an aquifer to the West and to the North of the reservoir and there are no injector wells.

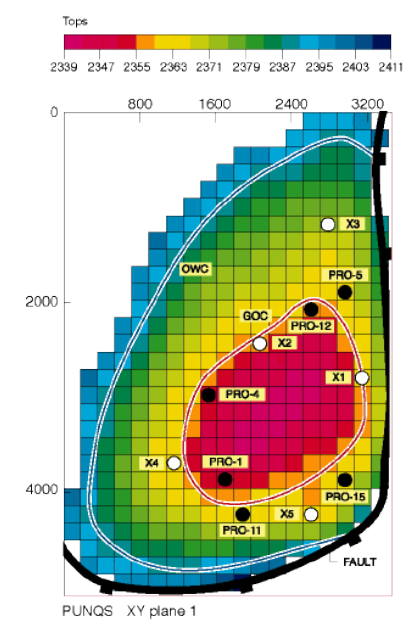

Figure 1: Colormap of the depth of the reservoir top, PUNQ-S3. 
In this HM problem, the vector of parameters $m$ consists of porosity and horizontal and vertical log-permeabilities at each grid cell. Geostatistical information about the parameters is given (basically, $C_{M}$ ), as well as measured values for these properties at well gridblocks (so-called hard data). This leaves us with about 5,200 parameters.

The history, consisting of bottom-hole pressure, water cut and gas-oil ratio at the wells, includes one year of extended well testing, followed by three years of field shut-in and four years of field production. The oil production rates for each well are given and used as boundary conditions, but if the minimum bottom-hole pressure for a well is reached, the simulation proceeds with such pressure prescribed.

Our PUNQ model displays some minor changes with respect to the original (see [2]) to accommodate for SIMPAR limitations. In our algorithm, we actually introduce a change of variables for the porosity, in order to generate an unconstrained optimization problem.

We have implemented RML to obtain a set of parameter fields honoring the geological a priori model, the hard data and the production history. Figure 2 shows the porosity fields of the true reservoir and of one solution obtained with TCG. They are quite different, as expected, since the HM problem has many solutions within the acceptable margin of error.

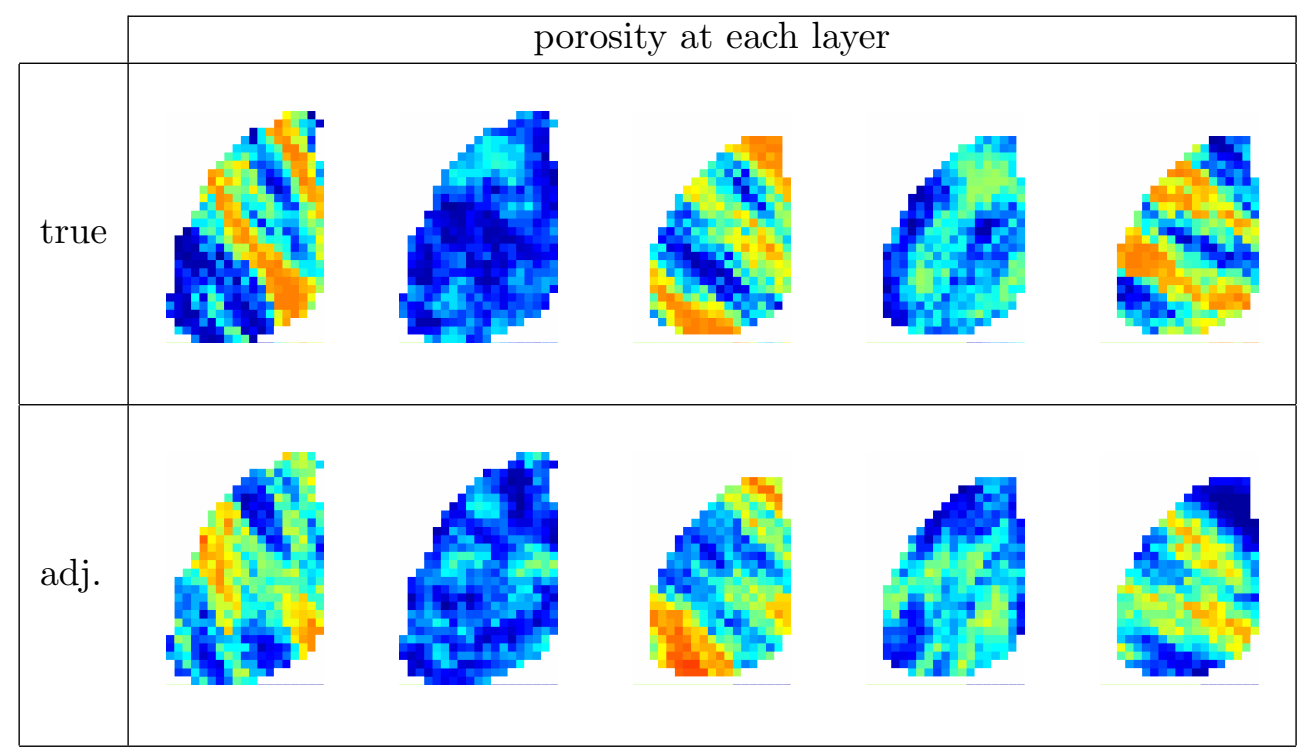

Figure 2: Porosity fields of the true and of one of the adjusted models.

We tested the ability of RML to predict the reservoir production. Figure 3 displays the graphs of the field cumulative productions of oil (left), gas (center) and water (right). Each green line represents the simulated production of one reservoir model obtained by use of TCG. To the left of the red line we have the 8-year history period, and to the right, the forecast for the following 8.5 years. The results for TSVD and L-BFGS are similar. We see that the true future behavior is contained in the fan of the extrapolations coming from RML. In those experiments, we consider that a simulation run has converged if $J\left(m_{\text {final }}\right) \leq D$. The value of $D$ we choose comes from probabilistic considerations, 
where the MAP solution is supposed to be a $\chi^{2}$ random variable, see $[8,11]$.
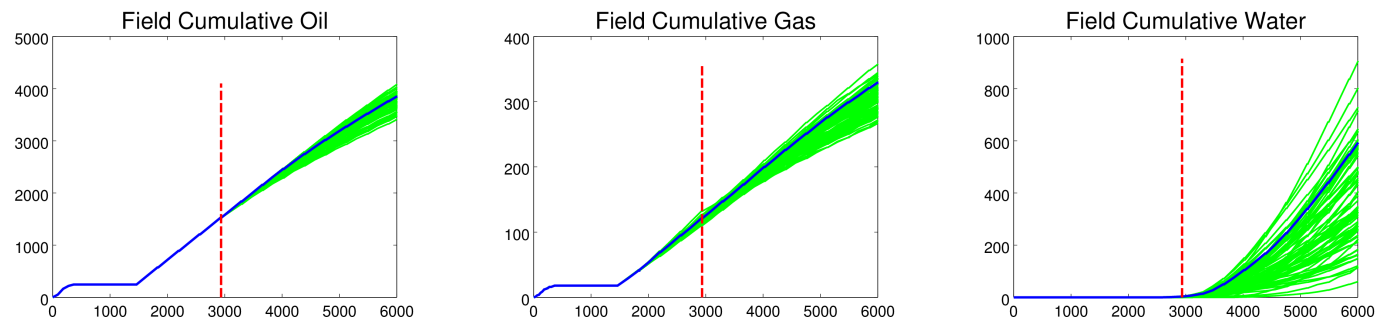

Figure 3: In green, extrapolations of cumulative oil, gas and water production. In blue, the true field. The red vertical line indicates the end of the history period.

Table 1 presents the total simulation time and the number of converged cases of TCG, TSVD and L-BFGS for 100 simulations using RML. TCG (n) indicates that at most $\mathrm{n}$ directions are used in each CG interation. We see that TSVD had a poor behavior when compared with the other schemes. While TCG was the most robust scheme, with the greatest number of converged runs, L-BFGS was the least time consuming. The total time divided by the number of successes is comparable for these two schemes. For others experiments in which TCG had clearly the best overall performance, see [2].

Table 1: Converged cases and time for 100 simulations.

\begin{tabular}{|c|c|c|}
\hline Algorithm & Converged cases & Total Time (sec.) \\
\hline L-BFGS & 35 & 33,843 \\
\hline TSVD & 46 & 209,860 \\
\hline TCG (5) & 79 & 73,254 \\
\hline TCG (10) & 78 & 99,725 \\
\hline
\end{tabular}

\section{Conclusion}

In this work we describe the TCG algorithm for History Matching. We used the standard benchmark PUNQ-S3 to compare TCG with TSVD and L-BFGS, two well known schemes for HM. In these experiments, TCG presented the most robust behavior, while L-BFGS displayed the smallest running time. We will develop further comparative studies on these schemes.

\section{Acknowledgement}

We are grateful to Petrobras for the support provided for the development of this work. Flávio Dickstein also acknowledges the partial support of CNPq. 


\section{References}

[1] F. Dickstein, P. Goldfeld, G. T. Pfeiffer, E. Amorim, R. W. dos Santos, and S. G. Gomez. A Study of the Impact of 4D-Seismic Data on TSVD-Based Schemes for History Matching. In Latin American and Caribbean Petroleum Engineering Conference 2010 (LACPEC 10), SPE 138864, 2010.

[2] F. Dickstein, P. Goldfeld, G. T. Pfeiffer, and R. Pinto, Truncated Conjugate Gradients for History Matching, to be submitted.

[3] Y. Dong and D. S. Oliver. Quantitative Use of 4D Seismic Data for Reservoir Description, SPE J. 10(1), 91-99, 2005. DOI:10.2118/84571-PA.

[4] G. Gao and A. C. Reynolds. An Improved Implementation of the LBFGS Algorithm for Automatic History Matching, SPE J. 11(1), 5-17, 2006. DOI:10.2118/90058-PA.

[5] G. H. Golub and C. F. van Loan. Matrix Computations (3rd ed.), The Johns Hopkins University Press, Baltimore, 1996.

[6] Imperial-College London, http://www.imperial.ac.uk/engineering/departments/earthscience/research/research-groups/perm/standard-models.

[7] J. Nocedal and S. J. Wright. Numerical Optimization (2nd ed.), Springer Series in Operations Research and Financial Engineering, Springer, New York, 2006. DOI:10.1007/978-0-387-40065-5.

[8] D. S. Oliver, A. C. Reynolds and N. Liu. Inverse Theory for Petroleum Reservoir Characterization and History Matching, Cambridge University Press, New York, 2008.

[9] D. W. Peaceman, Fundamentals of Numerical Reservoir Simulation, Elsevier, Amsterdam, 1977.

[10] J. R. P. Rodrigues. Calculating derivatives for automatic history matching, Computat. Geosci., 10(1), 119-136, 2006.

[11] A. Tarantola. Inverse Problem Theory and Methods for Model Parameter Estimation, SIAM, Philadelphia, 2005.

[12] R. Tavakoli and A. C. Reynolds. Monte Carlo simulation of permeability fields and reservoir performance predictions with SVD parameterization in RML compared with EnKF, Computat. Geosci., 15(1), 99-116, 2011.

[13] F. Zhang and A. C. Reynolds. Optimization Algorithms for Automatic History Matching of Production Data. In Proceedings of 8th European Conference on the Mathematics of Oil Recovery, Freiberg, 2002. 